\title{
The applications of androgen in the treatment of dry eye disease: a systematic review of clinical studies
}

\author{
Lixiang Wang and Yingping Deng \\ Department of Ophthalmology, West China Hospital, Sichuan University, Chengdu, 610041, People's Republic of China
}

\begin{abstract}
Androgen regulates the function of lacrimal and meibomian glands, and its deficiency is a pathological factor underlying dry eye disease (DED). However, no androgen has been approved for treating DED due to lack of definite evidence regarding its efficacy and safety in clinics. In this systematic review, we have summarized the clinical studies on the safety and efficacy of androgen replacement therapy (ART) for DED. Medline (via Pubmed), Embase, Clinicaltrials.gov, Wanfang and Chinese Clinical Trials Registry Database were searched for the relevant prospective studies, and 7 studies wherein androgen was applied topically via eye drops or systemically via oral or transdermal administration were included. The quality of these studies was assessed with the Cochrane Collaboration's tool for assessing risk of bias and methodological index for non-randomized studies. Most studies showed that androgen effectively improved dry eye-related symptoms and increased tear secretion. Furthermore, elderly men and peri-menopausal women with lower levels of circulating androgens responded better to ART. However, one study involving patients with Sjögren's syndrome showed no improvement in the ART group compared to the placebo control, or to the baseline level. Adverse effects were also common but limited to mild skin problems. In conclusion, androgen is a potential treatment for dry eye disease, especially for people with primary androgen deficiency. Short-term application is relatively safe.
\end{abstract}

Key words: Dry eye disease, Androgen, Replacement therapy

\section{Introduction}

Dry eye disease (DED) is a chronic condition characterized by instability of the tear film, and affects women and the elderly more often [1]. The prevalence of DED in the general population varies from $3 \%$ to $40 \%$ [2-7]. According to the 2013 National Health and Wellness Survey conducted on 75,000 adult individuals in USA, the prevalence of DED on the basis of subjective symptoms was $8.8 \%$ and $4.5 \%$ in women and men respectively [5]. Furthermore, a recent meta-analysis on the Chinese population reported a significantly higher prevalence of $31.4 \%$ based on self-reported symptoms [3]. Although most cases of DED involve only mild discomfort, it can progress to conjunctival injection, ocular surface inflammation, corneal neovascularization or even loss of vision [8]. In addition, the chronic discomfort associated with DED may lead to psychological problems such as depression [9]. It is also associated with a

Submitted Apr. 9, 2020; Accepted Aug. 3, 2020 as EJ20-0178 Released online in J-STAGE as advance publication Aug. 18, 2020 Correspondence to: Yingping Deng, Department of Ophthalmology, West China Hospital, Sichuan University, No. 37 Guoxue Alley, Wuhou District, Chengdu, 610041, China.

E-mail: ypd_wch@163.com considerable economic burden and causes an estimated loss of $\$ 3.84$ billion annually in USA alone [10].

Several in vitro and in vivo studies show that androgens increase tear secretion and osmolarity [11-13] upon binding to androgen receptors (AR) on the acinar cells of lacrimal glands $[14,15]$. They also play a vital role in lipid biosynthesis in the meibomian glands by regulating the expression of key genes associated with lipid uptake, chain elongation and lipid secretion [16-18]. Androgen deficiency is associated with meibomian gland dysfunction (MGD) and can lead to evaporative dry eye [13]. Given their role in maintaining both the lipid and aqueous components of the tear film, androgens are a potential therapeutic option in water-deficient as well as evaporative DED. Clinical studies show a higher risk of meibomian gland dysfunction and DED in patients with complete androgen-insensitivity syndrome (CAIS) or those on anti-androgen therapy [19-23]. In addition, elderly men and post-menopausal women with significantly lower levels of endogenous androgens are more susceptible to DED compared to younger individuals [24-26]. Since the serum androgen level correlates with lipid function and tear secretion [27, 28], androgen replacement therapy (ART) is a potential strategy to treat DED. However, due to the concerns of possible adverse effects 
and lack of approved androgen-based regimen for DED, androgens are not routinely used in the clinical setting. To this end, we conducted a systematic review of studies on the efficacy and safety of ART against DED.

\section{Methods}

\section{Data sources}

The literature search was conducted following the Preferred Reporting Items for Systematic Reviews and Meta-Analyses (PRISMA) guidelines [29]. The Medline (via Pubmed), Embase, Clinicaltrials.gov, Wanfang and Chinese Clinical Trials Registry Database were mined for clinical prospective studies on the application of ART for DED published in English and Chinese language till February 2nd, 2020 using the following MeSH key words: "dry eye syndrome", "dry eye disease", "keratoconjunctivitis sicca", "xerophthalmia", "eye dryness" and "androgen". The studies were independently screened by three investigators (Lixiang Wang, Jinqing Li and Shengqiang Zhang) who conducted a full test review of the selected articles. The detailed search strategy is outlined in the supplementary material. The final studies were included based on the following criteria: 1) randomized controlled trial (RCT), non-randomized trial, cohort study or self-controlled study, 2) including patients clinically diagnosed with DED, and 3) ART as the primary treatment for DED. Observational studies, case reports or retrospective studies, those involving combination therapy of androgens with other hormones and lacking an androgen monotherapy group, and animal studies were excluded.

\section{Quality assessment}

The quality of the selected RCTs was evaluated with Cochrane Collaboration's tool for assessing risk of bias [30] based on random sequence generation, allocation concealment, blinding of participants, personnel and outcome assessment, incomplete data, selective reporting and other biases. For non-randomized trials, methodological index for non-randomized studies (MINORS) was used [31].

\section{Data extraction}

The publication year, first author information, country, journal name, study type, number of participants, and the age and sex ratio were extracted from each study. In addition, outcome measures, such as TBUT, Schirmer's test, corneal fluorescence staining score, ocular surface disease index (OSDI) score, tear meniscus height, ocular comfort index, tear osmolality, serum testosterone level and adverse effects information were also extracted if available. Due to the high degree of heterogeneity among the studies in terms of study designs and outcome measures, a meta-analysis was not conducted.

\section{Results}

\section{Literature search}

A total of 1,297 studies were initially retrieved from Medline (147), Embase (296), Clinicaltrials.gov (740), Wanfang (67) and Chinese Clinical Trials Registry (47). After removing 107 duplicated studies, the abstracts of the remaining 1,190 studies were evaluated based on the inclusion and exclusion criteria. The full-text of 46 studies were reviewed, of which 7 were finally selected for the systematic review (Fig. 1). The characteristics of the eligible studies are summarized in Table 1. The assessments for risk of bias are listed in Figs. 2, 3 and Table 2.

\section{The effects of androgens on dry eye \\ (1) Transdermal administration}

Five studies, including 2 double-blind RCTs, used ointments containing $1-5 \%$ of testosterone to treat DED. Supalaset et al. recruited a total of 50 participants-

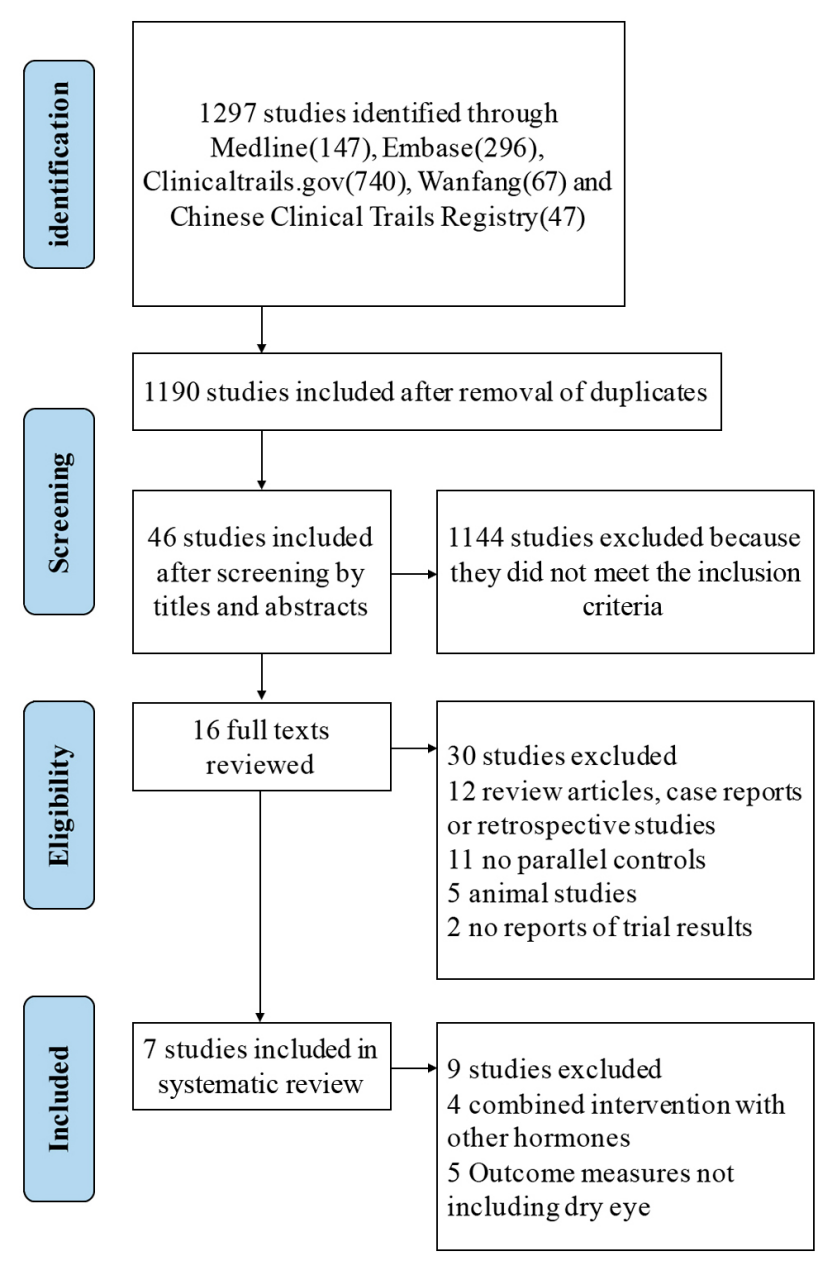

Fig. 1 Flow diagram for study selection 

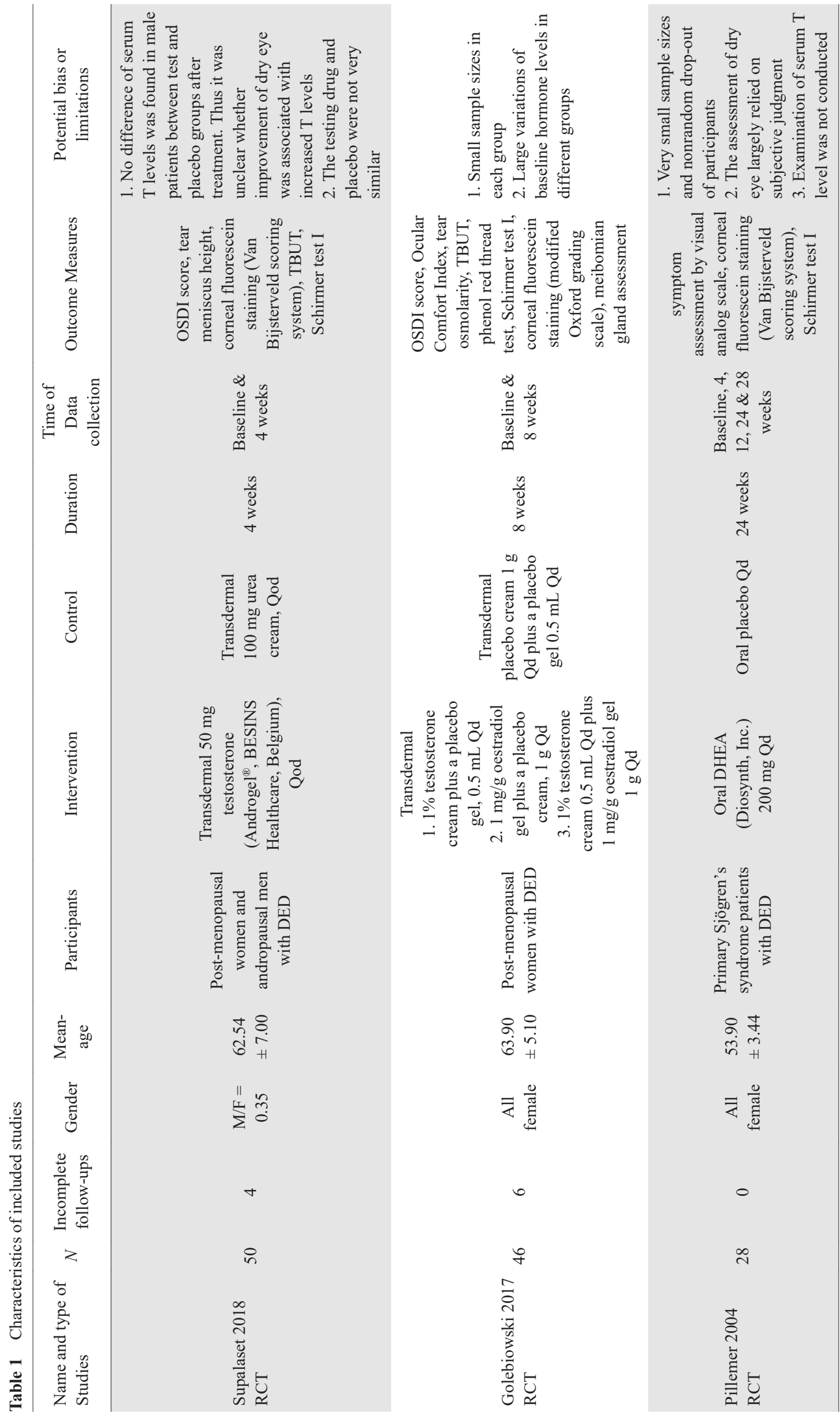


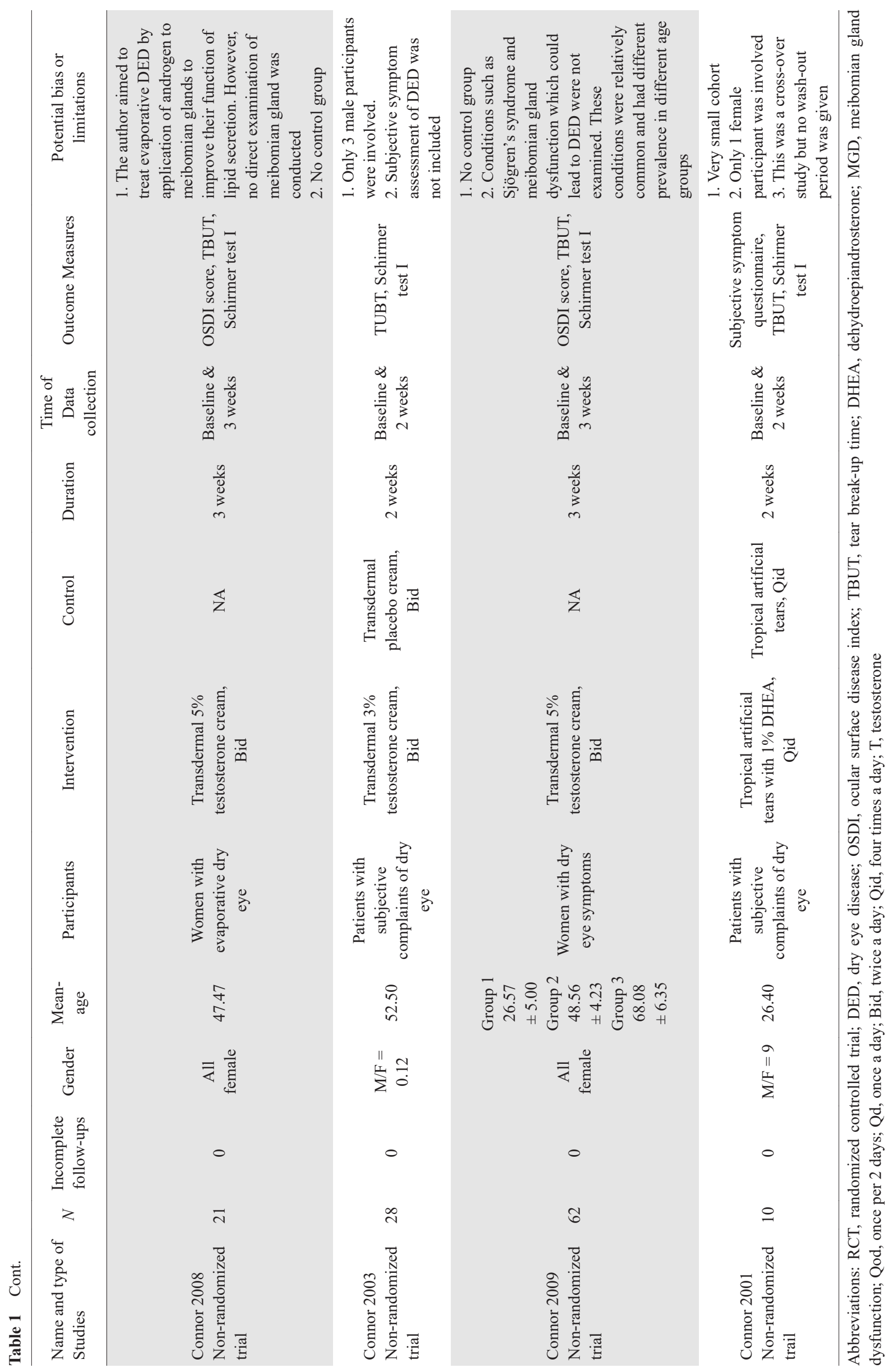




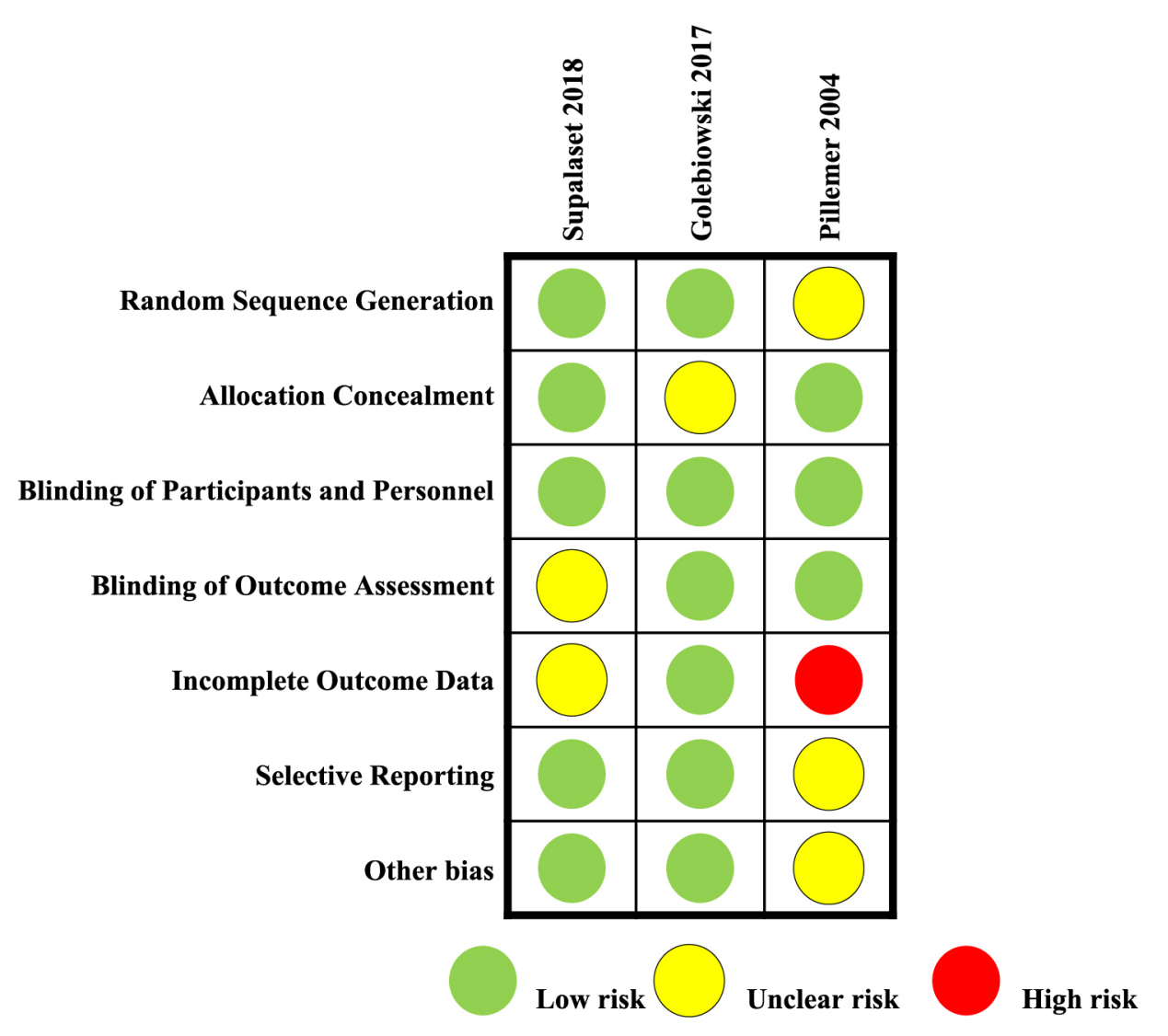

Fig. 2 Risk of bias of included RCTs by Cochrane Collaboration's tool for assessing risk of bias

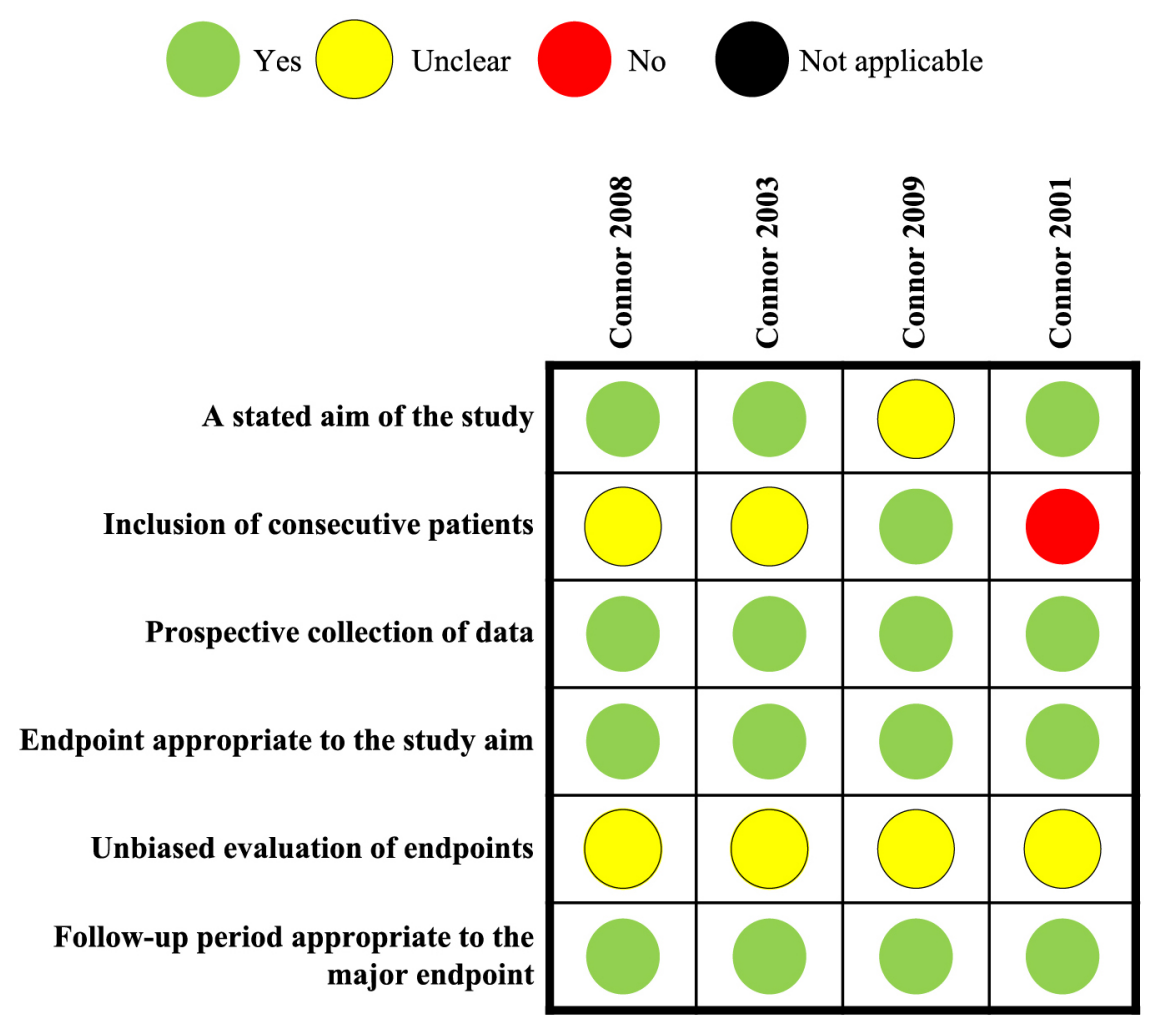

Fig. 3 Risk of bias of included non-randomized trials by methodological index for non-randomized studies (MINORS) 
Table 2 Risk of bias of included RCTs by Cochrane Collaboration's tool for assessing risk of bias (Only unclear and high risk items were listed in the table)

\begin{tabular}{|c|c|c|c|}
\hline Studies & Bias & $\begin{array}{l}\text { Author's } \\
\text { judgment }\end{array}$ & Support for judgment \\
\hline \multirow[t]{2}{*}{ Supalaset 2018} & $\begin{array}{l}\text { Blinding of } \\
\text { Outcome } \\
\text { Assessment }\end{array}$ & Unclear risk & $\begin{array}{l}\text { The author stated the study to be "double-masked" and described the } \\
\text { masking methods for patients, but didn't mention any detailed masking } \\
\text { methods for examiners. }\end{array}$ \\
\hline & $\begin{array}{l}\text { Incomplete } \\
\text { outcome data }\end{array}$ & Unclear risk & $\begin{array}{l}\text { The author stated "four patients in the placebo group were unable to attend a } \\
\text { follow-up". However, the author didn't evaluate how it affected the outcome. }\end{array}$ \\
\hline $\begin{array}{l}\text { Golebiowski } \\
2017\end{array}$ & $\begin{array}{l}\text { Allocation } \\
\text { concealment }\end{array}$ & Unclear risk & $\begin{array}{l}\text { The author stated "a random allocation sequence was generated using } \\
\text { Excel", but didn't mention about allocation concealment. }\end{array}$ \\
\hline \multirow{4}{*}{ Pillemer 2004} & $\begin{array}{c}\text { Random sequence } \\
\text { generation }\end{array}$ & Unclear risk & $\begin{array}{l}\text { The author stated "randomization resulted in } 14 \text { DHEA and } 14 \text { placebo group } \\
\text { subjects", but didn't mention any details about the method of random } \\
\text { sequence generation. }\end{array}$ \\
\hline & $\begin{array}{l}\text { Incomplete } \\
\text { outcome data }\end{array}$ & High risk & $\begin{array}{l}\text { The author stated "four DHEA and one placebo group patient dropped out } \\
\text { because of adverse effects". This number was substantial due to very small } \\
\text { total sample sizes. Besides, the author didn't mention whether the data from } \\
\text { previous visits of dropped-out patients were included in analysis. }\end{array}$ \\
\hline & Selective reporting & Unclear risk & $\begin{array}{l}\text { The author stated } 4 \text { visits at week } 4,12,24 \text { and } 28 \text { after enrollment, but only } \\
\text { the data from the last visit was reported. }\end{array}$ \\
\hline & Other bias & Unclear bias & $\begin{array}{l}\text { The author simply used visual analog scale to assess symptoms of dry eye } \\
\text { and dry mouth and didn't provide enough standards for participants to grade } \\
\text { their severity. A more thorough and verified questionnaire such as OSDI } \\
\text { score may be more appropriate. }\end{array}$ \\
\hline
\end{tabular}

menopausal women and andropausal men with low serum androgen levels - and instructed them to apply a cream containing either $50 \mathrm{mg}$ testosterone or $100 \mathrm{mg}$ urea on the lower abdomen on alternate days for 4 weeks. After 4 weeks of hormonal replacement, the OSDI score improved dramatically in the testosterone group, while no changes were reported in the placebo group. The quality of life (QOL) also improved for both male and female participants treated with testosterone. In addition, the TBUT increased by $7.4 \mathrm{~s}$ and the Schirmer's test result increased by $6.84 \mathrm{~mm}$ on average in the testosterone group. Other indices of dry eyes, including tear meniscus height and corneal fluorescein staining, also showed remarkable improvement in the testosterone versus placebo group [32]. Golebiowski et al. recruited 46 post-menopausal women and randomized them into the $1 \%$ testosterone, oestradiol, testosterone plus oestradiol and placebo groups. The participants were instructed to apply the test cream/gel once a day for 8 weeks. Both OSDI score and ocular comfort index (OCI) improved following testosterone administration but were not significant compared to the placebo group. Tear secretion, Schirmer's test results and TBUT also showed mild improvement in the testosterone group, whereas other clinical indices were similar to that of the placebo control. However, the serum level of the androgen metabolite $3 \alpha$-diol-G markedly increased in the testosterone group, and showed a strong positive correlation with the TBUT score [33].

Connor et al. conducted 3 studies to explore the efficacy of testosterone cream [34-36]. The studies were not blinded for participants or researchers, nor were the subjects randomly divided. The cohorts were small in three studies, and the participants were relatively younger and not necessarily androgen-deficient. In the first study, 28 participants (25 females and 3 males) applied either a placebo cream or $3 \%$ testosterone cream twice a day for 2 weeks. The Schirmer's test results increased significantly in the testosterone group compared with baseline and to the placebo group, while TBUT scores were similar in both groups. In addition, more than half of the testosterone users reported lessening of dry eye symptoms, and post-menopausal women exhibited maximum improvement. The testosterone dose was increased to $5 \%$ and the treatment regimen was prolonged to 3 weeks in the subsequent study on 21 female participants (22-68 years old) suffering from evaporative dry eye. The patients reported a significant relief of symptoms, with an average decrease of 15.94 in the OSDI score. TBUT also increased significantly after 3 weeks compared to the baseline. In the third study, 62 female DED patients were divided into 3 groups by age and treated via topical application of $5 \%$ testosterone cream on the eyelids. The women aged 40-60 years showed the greatest improvement after treatment, with the OSDI score dropping by $54.7 \%$, TBUT increasing by $2.48 \mathrm{~s}$ and Schirmer's test 
result increasing by $4.5 \mathrm{~mm}$ on average. Women aged under 40 and over 60 years only showed a slight improvement in symptoms and clinical signs.

\section{(2) Topical administration}

Connor et al. used eye drops containing 1\% dehydroepiandrosterone (DHEA), a precursor of testosterone, to treat DED [37] in 10 participants with subjective complaints of dry eye. All participants (including 9 young men) were instructed to use the test eye drops or placebo (artificial tears) four times a day for 2 weeks, and then switch to the other for another 2 weeks. All subjects reported irritation after using the DHEA eye drops, and $60 \%$ favored artificial tears with DHEA due to relief in dry eye symptoms. The TUBT and Schirmer's test results were significantly improved in the DHEA group compared with baseline and to the placebo group.

\section{(3) Oral administration}

Pillemer et al. conducted a double-blind randomized pilot study on 28 patients with primary Sjögren's syndrome wherein $200 \mathrm{mg}$ DHEA or placebo capsules were taken once a day for 24 weeks [38]. Previous studies have shown that the hypothalamic-pituitary-adrenal axis is inhibited in Sjögren's syndrome patients and decreases serum testosterone and DHEA levels [39, 40], resulting in an androgen-deficient state. The oral and ocular symptoms were subjectively assessed by the visual analog scale (VAS). No significant improvements were observed in the clinical symptoms, Schirmer's tests or corneal fluorescein staining compared with baseline and to the placebo group.

\section{Adverse effects}

Adverse effects were reported in 2 studies [38, 41]. Nine participants $(23.1 \%)$ in total reported adverse effects that may be associated with the ectopic androgen (Table 3). Most adverse reactions consisted of mild skin problems such as excessive oiliness of skin and acne, although 2 participants that took DHEA tablets reported more severe effects and discontinued the treatment. One participant with a history of recurrent acne suffered from a severe acne outbreak, and the other developed acute abdominal pain due to Streptococcus infection unrelated to DHEA. However, since both studies were conducted over a short period, the long-term adverse effects of ART are unknown.

\section{Discussion}

Although androgens promote lacrimal and meibum secretion, ART is not routinely used for treating DED due to lack of conclusive evidence regarding its efficacy and safety. In addition, no androgen formulation has been approved so far in China or USA for the indication of DED. Androgens can be administered through eye drops (topical), skin ointments and patches (transdermal), or pills (oral), and the application method greatly influence its bioavailability, efficacy and safety [42]. We conducted a systematic review of prospective studies analyzing the therapeutic effects of oral, transdermal and topical androgen formulations on DED. No other metaanalysis or systematic review has been published that summarizes the effects of androgen on DED symptoms. Only 7 studies met our inclusion criteria, of which 3 were RCTs. However, all studies had small sample sizes ranging from 10 to 62 participants (245 in total). While the RCTs were of relatively high quality with double blinding and randomization, the non-randomized clinical trials may have harbored additional bias from sample sizes, evaluation of outcome measures and the intervention of the parallel control group according to NIMORS standard.

Six studies showed that androgens administered via ointments or eye drops effectively relieved the symptoms of dry eye and increased tear stability over a period of 2 to 4 weeks. Furthermore, peri-menopausal women and elderly (andropausal) men with androgen deficiency responded best to the ART. The only study that did not show any ameliorative effects of androgen was conducted on patients with Sjögren's syndrome, a systemic autoimmune disease with excessive inflammatory cell

Table 3 List of adverse effects

\begin{tabular}{|c|c|c|c|c|c|}
\hline Form of androgen & $\begin{array}{c}\text { Way of } \\
\text { administration }\end{array}$ & Period & Adverse effects & $\begin{array}{l}\text { Androgen group } \\
\text { (total number) }\end{array}$ & $\begin{array}{l}\text { Placebo group } \\
\text { (total number) }\end{array}$ \\
\hline \multirow{2}{*}{ Testosterone } & \multirow{2}{*}{ Transdermal } & \multirow{2}{*}{4 weeks } & Oily skin & $5(25)$ & $0(21)$ \\
\hline & & & Acne & $1(25)$ & $0(21)$ \\
\hline \multirow{4}{*}{ DHEA } & \multirow{4}{*}{ Oral } & \multirow{4}{*}{24 weeks } & Severe acne & $1(14)$ & $0(14)$ \\
\hline & & & $\begin{array}{l}\text { Post-dose chills, } \\
\text { nervousness }\end{array}$ & $1(14)$ & $0(14)$ \\
\hline & & & $\begin{array}{c}\text { Disseminated } \\
\text { streptococcal infection }\end{array}$ & $1(14)$ & $0(14)$ \\
\hline & & & Perforated peptic ulcer & $0(14)$ & $1(14)$ \\
\hline
\end{tabular}


infiltration in the lacrimal glands that eventually leads to aqueous-deficient dry eye [43]. Even a 24-week treatment with DHEA did not improve self-reported symptoms or tear secretion. Therefore, ART can be considered for patients with primary deficiency of endogenous androgens but it may not be effective in patients with Sjögren's syndrome as a monotherapy.

Mild acne and oily skin were the most frequent adverse effects resulting from increased serum androgen levels, and diminished once ART was discontinued. One participant with a history of recurrent acne had a severe outbreak during the hormone treatment, and was thus likely sensitive to androgen. There are currently no reports on the adverse effects of long-term ART, which is of concern since DED is a chronic condition and may require a prolonged treatment. Besides, the frequently and easily noted skin problems related to androgen may break the blindness in the RCT studies.

In addition to the long-term safety and efficacy of ART, the time needed to reach maximum effects, the effect of the route of administration, potential sexassociated differences in treatment response, as well as objective assessments of DED need to be investigated further in order to establish evidence-based clinical androgen therapy for DED. There is currently no standard diagnostic criteria of DED, and its diagnosis and severity assessment mainly rely on self-reported symptoms, evaluation of tear secretion and signs of ocular surface damage [44]. Likewise, most studies included in this systematic review diagnosed DED on the basis of tear break-up time, corneal fluorescein staining score and subjective self-reported symptoms. Due to the inherently large inter- and intra-observer differences associated with such assays, an objective measurement scale is needed $[45,46]$. Furthermore, most studies only evaluated the aqueous secretion and ignored the effects on the lipid and mucus components of tears. A combination of noninvasive break-up time, objective automatic corneal staining, tear interferometer and tear chemokine and metallopeptidase levels can help assess DED in a more thorough and objective manner [44]. In addition, the accurate assessment of pre-treatment androgen levels is critical, especially for patients with suspected endogenous androgen deficiency, in order to select the correct participants and prevent adverse effects of supratherapeutic androgen levels [47]. Since the serum androgen level is influenced by gender, age, diurnal variations, intra-individual variations and detection method, the reference levels of normal serum androgen differ across laboratories $[48,49]$. In addition, the bioavailability and activity of androgen depends on the serum levels of binding proteins (mainly the sex hormone binding globulin or SHBG), activity of transferring enzymes
(5- $\alpha$ reductase) and the sensitivity of androgen receptors $[50,51]$. Therefore, it is also necessary to evaluate serum levels of androgen-related factors like total testosterone, free testosterone, dihydrotestosterone (DHEA) and SHBG at multiple time points throughout the regimen in order to monitor the therapeutic response. An adequate study duration is also needed to demonstrate the potential beneficial and harmful effects of androgen therapy. The time to reach the maximum effect of androgens should be determined via continuous evaluation of DED. Previous studies have reported considerable variation - ranging from 3 weeks to 12 months - in the time needed for androgen supplementation to show therapeutic effects due to factors like erection, depression, erythropoiesis and rise of prostate-specific antigens [52]. Since DED is a chronic condition requiring protracted therapy, longterm studies are needed in the future. Finally, the formulation and mode of administration also affect the outcome. Since ART was primarily designed to treat male patients with androgen deficiency, the appropriate doses for women and men with DED have not been standardized so far. Androgen can be administered via the oral, nasal, buccal, transdermal, subdermal and intramuscular routes, which influence the acting time (short for buccal, nasal and topical routes, and long for intramuscular and subdermal routes), dosing frequency, bioavailability (high for intramuscular injection and low for topical eye drops) as well as formulation-specific adverse effects (for e.g. gingivitis for buccal tablets and skin blistering for skin patches) [53]. More studies are needed to compare their efficacy and adverse effects, and therefore choose the more appropriate forms for DED.

This is the first systematic review which addresses the effects of androgen therapy via oral, transdermal and topical applications on DED. The studies included in this review demonstrated the therapeutic effects of androgen on male and female patients with endogenous androgen deficiency, as well as younger individuals with normal androgen levels. However, there are some limitations in our study that ought to be addressed. First, only 3 RCTs were included and all studies had small sample sizes. Furthermore, insufficient data prevented further metaanalysis to obtain conclusive results. Second, the study durations of most studies were $2-4$ weeks, which was insufficient to observe the long-term adverse effects of androgen therapy. Finally, there was potential risk of bias from the selection of studies as all 4 non-RCTs were reported by the same researcher and only 7 useful studies were obtained. Therefore, more studies with larger sample sizes need to conducted in the future. 


\section{Conclusions}

Short-term ART is a potential and relatively safe option for patients with DED, especially for those with primary androgen deficiency.

\section{Acknowledgement}

We thank Jinqing Li and Shengqiang Zhang of the
Sichuan University Library for their kind assistance in literature search.

\section{Disclosure}

None of the authors have any potential conflicts of interest associated with this research. The authors alone are responsible for the content and writing of the paper.

\section{Reference}

1. Pflugfelder SC (2008) Prevalence, burden, and pharmacoeconomics of dry eye disease. Am J Manag Care 14: S102-S106.

2. Titiyal JS, Falera RC, Kaur M, Sharma V, Sharma N (2018) Prevalence and risk factors of dry eye disease in North India: ocular surface disease index-based crosssectional hospital study. Indian J Ophthalmol 66: $207-$ 211.

3. Song P, Xia W, Wang M, Chang X, Wang J, et al. (2018) Variations of dry eye disease prevalence by age, sex and geographic characteristics in China: a systematic review and meta-analysis. J Glob Health 8: 020503.

4. Alshamrani AA, Almousa AS, Almulhim AA, Alafaleq AA, Alosaimi MB, et al. (2017) Prevalence and risk factors of dry eye symptoms in a Saudi Arabian population. Middle East Afr J Ophthalmol 24: 67-73.

5. Farrand KF, Fridman M, Stillman IO, Schaumberg DA (2017) Prevalence of diagnosed dry eye disease in the United States among adults aged 18 years and older. $A m J$ Ophthalmol 182: 90-98.

6. Yen JC, Hsu CA, Li YC, Hsu MH (2015) The prevalence of dry eye syndrome's and the likelihood to develop Sjögren's syndrome in Taiwan: a population-based study. Int J Environ Res Public Health 12: 7647-7655.

7. Schaumberg DA, Dana R, Buring JE, Sullivan DA (2009) Prevalence of dry eye disease among US men: estimates from the physicians' health studies. Arch Ophthalmol 127: 763-768.

8. Bron AJ, de Paiva CS, Chauhan SK, Bonini S, Gabison EE, et al. (2017) TFOS DEWS II pathophysiology report. Ocul Surf 15: 438-510.

9. Jonas JB, Wei WB, Xu L, Rietschel M, Streit F, et al. (2018) Self-rated depression and eye diseases: the beijing eye study. PLoS One 13: e0202132.

10. Yu J, Asche CV, Fairchild CJ (2011) The economic burden of dry eye disease in the United States: a decision tree analysis. Cornea 30: 379-387.

11. Song X, Zhao P, Wang G, Zhao X (2014) The effects of estrogen and androgen on tear secretion and matrix metalloproteinase-2 expression in lacrimal glands of ovariectomized rats. Invest Ophthalmol Vis Sci 55: 745751.

12. Luo F, Zhang H, Sun X (2001) The change of tear secre- tion and tear film stability in castrated male rabbits Zhonghua Yan Ke Za Zhi 37: 458-461 (In Chinese).

13. Krenzer KL, Dana MR, Ullman MD, Cermak JM, Tolls DB, et al. (2000) Effect of androgen deficiency on the human meibomian gland and ocular surface. J Clin Endocrinol Metab 85: 4874-4882.

14. Gao J, Lambert RW, Wickham LA, Banting G, Sullivan DA (1995) Androgen control of secretory component mRNA levels in the rat lacrimal gland. J Steroid Biochem Mol Biol 52: 239-249.

15. Sullivan DA, Kelleher RS, Vaerman JP, Hann LE (1990) Androgen regulation of secretory component synthesis by lacrimal gland acinar cells in vitro. J Immunol 145: 42384244.

16. Rocha EM, Wickham LA, da Silveira LA, Krenzer KL, Yu FS, et al. (2000) Identification of androgen receptor protein and 5alpha-reductase mRNA in human ocular tissues. Br J Ophthalmol 84: 76-84.

17. Driver PJ, Lemp MA (1996) Meibomian gland dysfunction. Surv Ophthalmol 40: 343-367.

18. Sullivan DA, Jensen RV, Suzuki T, Richards SM (2009) Do sex steroids exert sex-specific and/or opposite effects on gene expression in lacrimal and meibomian glands? Mol Vis 15: 1553-1572.

19. Sullivan DA, Sullivan BD, Evans JE, Schirra F, Yamagami H, et al. (2002) Androgen deficiency, meibomian gland dysfunction, and evaporative dry eye. Ann $N Y$ Acad Sci 966: 211-222.

20. Sullivan DA (2004) Androgen deficiency \& dry eye syndromes. Arch Soc Esp Oftalmol 79: 49-50.

21. Sullivan DA, Yamagami H, Liu M, Steagall RJ, Schirra F, et al. (2002) Sex steroids, the meibomian gland and evaporative dry eye. Adv Exp Med Biol 506: 389-399.

22. Cermak JM, Krenzer KL, Sullivan RM, Dana MR, Sullivan DA (2003) Is complete androgen insensitivity syndrome associated with alterations in the meibomian gland and ocular surface? Cornea 22: 516-521.

23. Sullivan BD, Evans JE, Cermak JM, Krenzer KL, Dana MR, et al. (2002) Complete androgen insensitivity syndrome: effect on human meibomian gland secretions. Arch Ophthalmol 120: 1689-1699.

24. Sriprasert I, Warren DW, Mircheff AK, Stanczyk FZ (2016) Dry eye in postmenopausal women: a hormonal 
disorder. Menopause 23: 343-351.

25. Yasui T, Matsui S, Tani A, Kunimi K, Yamamoto S, et al. (2012) Androgen in postmenopausal women. J Med Invest 59: $12-27$.

26. Grossmann M, Wu FC (2014) Male androgen deficiency: a multisystem syndrome. Asian J Androl 16: 159-160.

27. Azcarate PM, Venincasa VD, Feuer W, Stanczyk F, Schally AV, et al. (2014) Androgen deficiency and dry eye syndrome in the aging male. Invest Ophthalmol Vis Sci 55: 5046-5053.

28. Truong S, Cole N, Stapleton F, Golebiowski B (2014) Sex hormones and the dry eye. Clin Exp Optom 97: 324-336.

29. Moher D, Shamseer L, Clarke M, Ghersi D, Liberati A, et al. (2015) Preferred reporting items for systematic review and meta-analysis protocols (PRISMA-P) 2015 statement. Syst Rev 4: 1 .

30. Higgins JP, Altman DG, Gøtzsche PC, Jüni P, Moher D, et al. (2011) The Cochrane Collaboration's tool for assessing risk of bias in randomised trials. BMJ 343: d5928.

31. Dent $O$ (2003) Methodological index for non-randomized studies. ANZ J Surg 73: 675-676.

32. Supalaset S, Tananuvat N, Pongsatha S, Chaidaroon W, Ausayakhun S (2019) A randomized controlled doublemasked study of transdermal androgen in dry eye patients associated with androgen deficiency. Am J Ophthalmol 197: 136-144.

33. Golebiowski B, Badarudin N, Eden J, Gerrand L, Robinson J, et al. (2017) The effects of transdermal testosterone and oestrogen therapy on dry eye in postmenopausal women: a randomised, placebo-controlled, pilot study. Br J Ophthalmol 101: 926-932.

34. Connor CG (2003) Treatment of dry eye with a transdermal 3\% testosterone cream. IOVS 44: 2450.

35. Connor CG (2008) Evaporative dry eye treated with transdermal testosterone. IOVS 49: 120.

36. Connor CG (2009) Androgenic cream treatment most efficacious for women 40 to 60 with dry eye. IOVS 50: 4656.

37. Connor C, Karkkainen T (2001) The efficacy of androgenic artificial tears in the treatment of dry eye. Scientific Program of 78th Annual Meetings of American Academy of Optometry, R114-3: 40 pm (Abstract).

38. Pillemer SR, Brennan MT, Sankar V, Leakan RA, Smith JA, et al. (2004) Pilot clinical trial of dehydroepiandrosterone (DHEA) versus placebo for Sjögren's syndrome. Arthritis Rheum 51: 601-604.

39. Valtysdóttir ST, Wide L, Hällgren R (2001) Low serum dehydroepiandrosterone sulfate in women with primary Sjögren's syndrome as an isolated sign of impaired HPA axis function. J Rheumatol 28: 1259-1265.

40. Valtysdottir ST, Wide L, Hallgren R (2003) Mental wellbeing and quality of sexual life in women with primary Sjögren's syndrome are related to circulating dehydro- epiandrosterone sulphate. Ann Rheum Dis 62: 875-879.

41. Supalaset S, Tananuvat N, Pongsatha S, Chaidaroon W, Ausayakhun S (2018) A randomized controlled doublemasked study of transdermal androgen in dry eye patients associated with androgen deficiency. Am J Ophthalmol 197: 136-144.

42. Laurent MR, Helsen C, Antonio L, Schollaert D, Joniau S, et al. (2016) Effects of sex hormone-binding globulin (SHBG) on androgen bioactivity in vitro. Mol Cell Endocrinol 437: 280-291.

43. Thorne I, Sutcliffe N (2017) Sjögren's syndrome. Br J Hosp Med (Lond) 78: 438-442.

44. Wolffsohn JS, Arita R, Chalmers R, Djalilian A, Dogru M, et al. (2017) TFOS DEWS II diagnostic methodology report. Ocul Surf 15: 539-574.

45. Tong L, Teng LS (2018) Review of literature on measurements of non-invasive break up times, lipid morphology and tear meniscal height using commercially available hand-held instruments. Curr Eye Res 43: 567-575.

46. Sook Chun Y, Park IK (2014) Reliability of 4 clinical grading systems for corneal staining. Am J Ophthalmol 157: 1097-1102.

47. Trost LW, Mulhall JP (2016) Challenges in testosterone measurement, data interpretation, and methodological appraisal of interventional trials. J Sex Med 13: 10291046.

48. Diver MJ, Imtiaz KE, Ahmad AM, Vora JP, Fraser WD (2003) Diurnal rhythms of serum total, free and bioavailable testosterone and of SHBG in middle-aged men compared with those in young men. Clin Endocrinol (Oxf) 58: 710-717.

49. Bhasin S, Pencina M, Jasuja GK, Travison TG, Coviello A, et al. (2011) Reference ranges for testosterone in men generated using liquid chromatography tandem mass spectrometry in a community-based sample of healthy nonobese young men in the Framingham Heart Study and applied to three geographically distinct cohorts. J Clin Endocrinol Metab 96: 2430-2439.

50. Dhiman P, Bhansali A, Prasad R, Dutta P, Walia R, et al. (2011) Predictors of pilosebaceous unit responsiveness to testosterone therapy in patients with hypogonadotrophic hypogonadism. Andrologia 43: 422-427.

51. Pardridge WM (1986) Serum bioavailability of sex steroid hormones. Clin Endocrinol Metab 15: 259-278.

52. Saad F, Aversa A, Isidori AM, Zafalon L, Zitzmann M, et al. (2011) Onset of effects of testosterone treatment and time span until maximum effects are achieved. Eur $J$ Endocrinol 165: 675-685.

53. Shoskes JJ, Wilson MK, Spinner ML (2016) Pharmacology of testosterone replacement therapy preparations. Transl Androl Urol 5: 834-843. 